\title{
Round Table Summary: Problems in Modelling Stellar Atmospheres
}

\author{
Fiorella Castelli \\ Istituto di Astrofisica Spaziale e Fisica Cosmica, CNR, Via del Fosso \\ del Cavaliere, 00133, Roma, Italy
}

The aim of the Round Table (RT) "Problems in modelling stellar atmospheres" was that of giving a panoramic view of the state of art on the atmospheric models used to predict observations of stars from $\mathrm{T}$ to A spectral type, the Sun included.

To this purpose the managers of the session "Current status of modelling", Fiorella Castelli and Peter Hauschildt, had invited some people on the floor, on the basis of all the abstracts made available to them before the Conference. Selected people were asked for a short presentation $(4 \mathrm{~min})$ of their posters.

People on the floor were: A.C. Andersen, E. H. Avrett, K. Eriksson, D. Homeier, N. Nesvacil, Y. Pavlenko, and B. Plez. In addition, also B. Baron, S. Dreizler, B. Gustaffson, and R.L. Kurucz were asked by the IAU Symposium organizers to mount on the floor, as they had an invited talk in that session. P. Hauschildt, who did not attend to the conference, was replaced as co-chairman of the RT by B. Gustaffson. The allocated time for the RT was 1 hour. follows.

Starting from the coolest stars, the sequence of the presentations was as

A. C. Andersen (poster A13) pointed out the importance of modelling dust grains to better understand mass loss in asymptotic giant branch (AGB) stars; in particular she showed the influence of dust grains on the wind of carbon-rich AGB stars. The codes of the Höfner group (Session F in this volume) were used for this research.

D. Homeier (poster E56) presented model atmospheres, including dust, well suited to study $\mathrm{T}$-type dwarfs $\left(\mathrm{T}_{\text {eff }} \leq 1400 \mathrm{~K}\right)$. These models were generated by the PHOENIX code (Baron et al., Session A in this volume). Particular attention was paid to the opacity of $\mathrm{CH}_{4}$, which strongly affects the IR spectrum of these very cool stars.

K. Eriksson (poster A4) presented an upgraded extended grid of more than 5000 MARCS models.

B. Plez presented a study of S-type stars $\left(3000 \leq \mathrm{T}_{\text {eff }} \leq 4000 \mathrm{~K}\right)$ performed by using a subset of the new MARCS models. The effects of the $\mathrm{ZrO}$ bands and of different $\mathrm{C} / \mathrm{O}$ ratios were pointed out.

E.H. Avrett (poster A21) presented the upgrades of the PANDORA code, which computes NLTE models including the effects of mass flows. PANDORA is primarely well suited to study the outer atmosphere of the Sun and other late-type stars.

Y. Pavlenko (poster A16) showed comparisons of predictions with observations for some extreme chemically peculiar stars (R CrB-like stars, Sakurai's object (V4334 Sgr), Przybylski's star), which span a range in $\mathrm{T}_{\text {eff }}$ from $4000 \mathrm{~K}$ 
to $6500 \mathrm{~K}$. Computations were made with the opacity sampling code SAM12, which is a modification of the ATLAS12 code from Kurucz. Some emphasis was given to the most important opacity sources acting in the studied stars.

Finally, N. Nesvacil (poster A9) presented a procedure to compute color indices from grids of ATLAS9 models, which were generated in Vienna and Paris by modifying some of the input parameters adopted by Kurucz.

A discussion between people on the floor and the participants to the conference has followed. The discussion was mostly related with the improvements in the models, the differences between different sets of models, the availability of new atomic and molecular line lists, and the several future improvements expected in the field. However, because the invited presentations were too many for the allocated time, the discussion stopped short time after it started. 